\title{
A Comparative Study of Controlling A Pneumatic Conveying System with a Multifunctional Interface and Simulation
}

\author{
Michail Papoutsidakis, Dimitrios Tseles, Pantelis Malatestas \\ Department of Automation Engineering \\ Piraeus University of Applied Sciences \\ Athens, Greece
}

\begin{abstract}
Modern servopneumatic positioning technology has made significant inroads in the automated manufacturing environment. The advantages cited by end users include the speed of motion, low cost of installation and maintenance, cleanliness, and the simplicity of operation of these systems relative to other similar hydraulic and electro-mechanical technologies. The robustness of servopneumatic technology solutions is limited by the positioning accuracy of current system controllers. Servopneumatic controllers typically rely on sophisticated control algorithms that accommodate the highly non-linear nature of pneumatic actuator operation. Taken as a whole, these system characteristics provide an ideal modern laboratory setup for instruction in the use of positioning controllers and the development of supporting control methodologies. In this research work, an attempt to control a pneumatic actuator in a real rig simultaneously with a simulation model, is proposed. Two different control methods were chosen for the task, a auto-selective format of PID control and a fuzzy logic based one. The mathematical model of the system will be provided and the comparison results of operation are illustrated in the paper supporting the efficiency of the two applied control methods. Additionally, an embedded electronics board was designed and constructed to fulfill successfully the interface needs. On the board, a microcontroller was mounted and all its necessary peripherals in order to host the control algorithms, communicate with the pneumatic plant and at the same time operate as a data acquisition system.
\end{abstract}

\section{Introduction}

Due to their high power-to-weight ratio and low cost, pneumatic actuators are attractive for robotics and automation applications; however, achieving fast and accurate control of their position have been known as a complex control problem. A methodology for obtaining high position accuracy with a linear pneumatic actuator is presented. During experimentation with a number of PID classical control approaches over many operations of the pneumatic system, the need for frequent manual retuning of the controller could not be eliminated. The reason for this problem is thermal and energy losses inside the cylinder body due to the complex friction forces developed by the piston displacements see [1], [2] and [3]. Although PD controllers performed very well over short periods, it was necessary in this research project to introduce some form of autoselective use of the control terms and achieve the ultimate system results in the position-tracking problem of a servo pneumatic system applying a traditional PD controller only in the first step of the controller design. The use of the I-term control will be selective if the system performance requires although it will be included in the control algorithm from the beginning. There is a significant number of experimentation and well presented results already referring to fuzzy logic controllers applied to pneumatic systems, see [4] and [5]. This fact keeps encouraging researchers to study deeper the area of controlling pneumatic control with fuzzy logic controllers and therefore will be implemented in this project as well. It was considered as a nice idea to investigate deeper the performance of the specific system under a fuzzy controller and prove even further the versatility and multi-functionality of the proposed interface board. At the top of all these, the most important part of the system is an interface board which will host the controller and all electronics peripherals. The interface card will be designed and assembled in order to be used for long term operations of the system. The criteria for choosing these specific microprocessor and electronics, details are provided in the following paragraphs, are low cost, reliability and multitasking capabilities. The ease of use in terms of programming was taken under consideration since the multiple input positioning system requires long control algorithms to be compiled by the microcontroller. One of the interface board main purposes is also data acquisition and real time data monitoring on a computer screen. In this paper, the 
pneumatic positioning system description and the control method implementation are given in the first paragraphs. The electronic interface board parts and assembly details will follow and finally the results and some further future applications will be discussed.

\section{System Description}

The pneumatic positioning system under investigation consists of a double acting pneumatic cylinder (type DSW-32-80PPV-A), stroke of $80 \mathrm{~mm}$, combined with a pneumatic proportional control servo-valve (type MPYE-5-1/8). The controller of the plant will have to read the current position of the pneumatic piston and correct the input of the system in order to minimize the error. The position sensor is a Linear Variable Differential Transducer (LVDT) and pressure sensors are also included in the system to increase the performance of the controller, by providing more data to it. The main layout of the system with all its parts connected is provided in fig 1. It is obvious in the figure that the inputs of the system are the actual piston position provided by the LVDT and the two different air pressure values given by the pressure sensors. The output of it is the control signal in Volts produced by the microprocessor as a result of the control algorithm implementation that drives the servo-valve. The last one is the control device of the plant, which operates the whole system under the controller's commands. The valve opens and closes its ports in order to compress or decompress air in the two cylinder chambers and therefore move the piston.

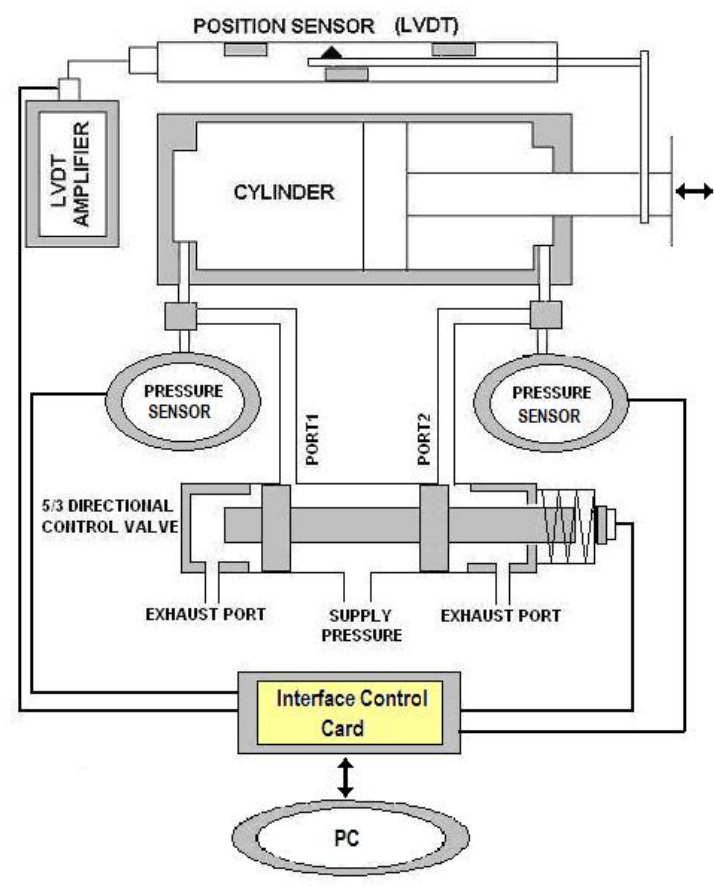

Figure 1. The system main layout
The MPYE-5- proportional valve is a 5/3-way function distributor. It is thus composed of five ports $\mathrm{N}, \mathrm{P}, \mathrm{S}, \mathrm{E}$ and $\mathrm{E}$ with three configurations for the air flow (Figure 2). The five ways present an $S$ orifice of compressed air (it is numbered orifice (1) by the manufacturer), two working orifices for $\mathrm{P}(4), \mathrm{N}(2)$ and two for the exhaust E(3 and 5). With the MPYE valve, the valve-slide stroke is controlled proportionally to a specified set point, as in [6]. The analog electrical input signal to the MPYE produces a step less variation of the flow rate. The MPYE thus controls the flow rate regard its magnitude and direction.

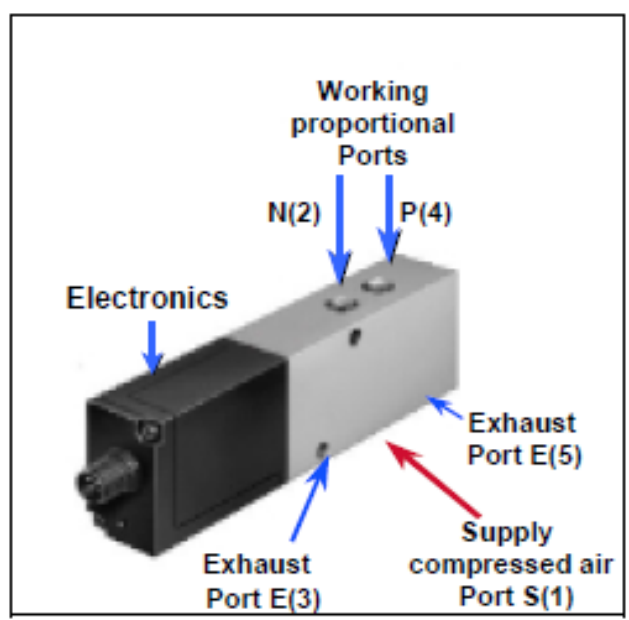

Figure 2. The MPYE valve schematics

The MPYE valve operates between 0 and $10 \mathrm{~V}$. At $5 \mathrm{~V}$ input, the flow rate is disabled. As the voltage input is decreased from 5 to $0 \mathrm{~V}$, the flow rate increases in the negative direction. As the input voltage is increased from $5 \mathrm{~V}$ to $10 \mathrm{~V}$, the flow rate increases in the positive direction. Figure 3 below illustrates the output of the MPYE proportional control valve. The potentiometer outputs a voltage proportional to the position of the slide. The potentiometer operates between 0 and $10 \mathrm{~V}$. As the flow rate into and from the cylinder is varied through the MPYE valve, the pressure on either side of the piston in the cylinder is varied. Resulting force imbalance causes motion of the piston. For a voltage of $5 \mathrm{~V}$, when the spool is in mid-position, the flow rate of the distributor is theoretically null. If the input control is varied from $5 \mathrm{~V}$ to $0 \mathrm{~V}$, the compressed air exits from port (1) towards port (2) and the exhaust goes from port (4) towards port (5). By varying the voltage of $5 \mathrm{~V}$ to $10 \mathrm{~V}$, the compressed air exits from port (1) towards port (4) and the exhaust goes from port (2) towards port (3).

The characterization suggested for the FESTO proportional valve is a global static characterization for constant exhaust and supply pressures.

- Global since the output mass flow rate is given as a function of both the electrical 
proportional valve input and of the output pressure, the supply and exhaust ports being as in normal working conditions which means that none of

- Btanincis bletause for each variation in the electrical input control or in the output working pressure, the measurement readings (pressure and flow rate) are taken when the flow rate is in established mode.

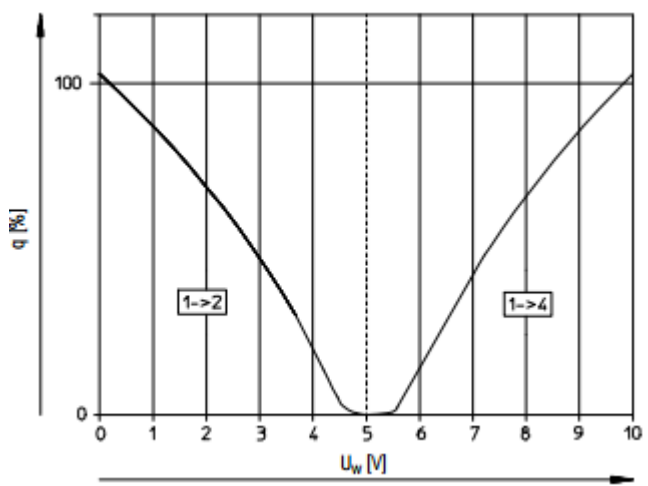

Figure 3. Flow rate $\mathrm{q}$ at 6 bar as a function of the setpoint voltage $U$

At this point the difficult part of the system dynamics and controlling occur, since the air compression is a highly nonlinear phenomenon. The mathematical model of the pneumatic cylinder and the valve consists of third order system equations and in fact they are also switching depending on the air pressure. All mathematics and system modeling can be found in [7] and [8]. For illustration purposes some mathematical equations will be provided at this point, which will be included in the simulation blocks of the system, as described later. The interesting part of this analysis is focused on the servo valve dynamics, regarding air mass flow rate $(Q)$ :

$Q=\left\{\begin{array}{l}C_{f} A i C_{1} P_{1} / \sqrt{T}, \\ \text { if } P_{2}>P_{1} P_{C r} \\ C_{f} A i C_{2} P_{2} /(\sqrt{T})\left(\frac{P_{2}}{P_{1}}\right)\left(\frac{1}{K}\right) \sqrt{1-\left(\frac{P_{2}}{P_{1}}\right)\left(\frac{K-1}{K}\right)} \\ \text { if } P_{2} \leq P_{1} P_{C r}\end{array}\right.$,

Where,

Cf, $\mathrm{C}_{1}, \mathrm{C}_{2}, \mathrm{~K}$, are constant coefficients, $\mathrm{Ai}$ is the valve orifice area, $\mathrm{P}_{1}$ and $\mathrm{P}_{2}$ are the air pressures entering the two cylinder chambers and $\mathrm{P}_{\mathrm{cr}}=0.528$ is the critical pressure value separating the supersonic from the subsonic circumstances of the system operation.

According to the same authors [7] and [8] the pressure derivative build-up equations inside each of the two cylinder chambers is shown below:

$$
P_{1,2}^{o}=\left[R T Q_{1,2}-\alpha P_{1,2} A_{1,2} x\right] / A_{1,2}(L-x)
$$

Where,

$$
\text { o }
$$

$x$ the piston velocity, $\mathrm{x}$ is the piston position, $\mathrm{T}$ is the temperature, $\mathrm{R}$ is the ideal gas constant, $\mathrm{A}_{1,2}$ is the piston's areas from both sides, $\mathrm{L}$ is the piston stroke, $\alpha$ is a coefficient depending on the heat transfer and their values can be easily calculated and for simplicity reasons reporting them is neglected here. The equation of the piston motion is derived from the second Newton's Law:

$$
00
$$

$$
P_{1} A_{1}-P_{2} A_{2}-M g-F f-b x=M x
$$

Where,

OO

$x$ the piston acceleration, $M$ the mass of the load, $\mathrm{g}$ the gravitational force (the cylinder is placed vertically), $\mathrm{P}_{1}$ and $\mathrm{P}_{2}$ the chambers pressures, $\mathrm{b}$ is the spring constant and $\mathrm{A}_{1}, \mathrm{~A}_{2}$ the piston area in each chamber respectively, $\mathrm{Ff}$ the combined static and viscous friction force. It must be stated that $100 \%$ position accuracy of any pneumatic piston has not be achieved yet and therefore there is still area for more research like the one in this project.

The simulation model of the overall system was designed and implemented in Matlab ${ }^{\circledR}$ Simulink ${ }^{\circledR}$ software environment. The PID part of the modelling is shown below in Figure 4. At this point, it must be stated that the simulation experimentation of the pneumatic system is a complementary work of a real pneumatic rig set up as shown in [9]. Based on the results coming out from the real world control applications in the same system, it is more informative to compare and check the simulation model outcomes and understand whether or not the simulation model represents the real system. The outcomes of such a simulation as well as comparisons of the real system behaviour are provided later in this paper. 


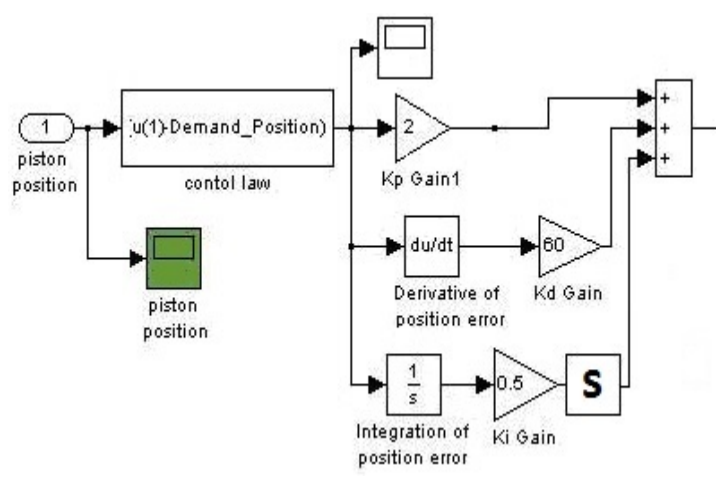

Figure 4. The simulation blocks of the controller

\section{The Interface Board}

In order to be able to control and record the behavior of the pneumatic plant, an interface card was designed and assembled. This should be able to convert the analog and continuous signal into digital words and also the digital input into an analog control signal to drive the pneumatic servo valve. Criteria like low cost electronics and multiple power supplies for the microcontroller and its peripheral equipment should be kept. The pneumatic valve response time is $50 \mathrm{msec}$ so the interface card should be faster than that to drive the valve properly. The controller reads the current position of the pneumatic piston and corrects the input of the system according to the control algorithm in order to minimize the error. Pressure sensors are also available to increase the performance of the controller. The first sensor, the LVDT, produces an analog signal of 0-5 Volt. An Analog to Digital Converter is used, specified to the highest accuracy for such a signal. The pressure sensor, varies between 0-10Volts, so the Analog to Digital converter needs to be circuited a different way, to produce the maximum accuracy. The valve on the other hand, moves up when the input is 0 - 5Volts, which is shown in Figure 5.

If its input is $5-10$ Volts then it moves down. At the exact input of 5Volts there is no action. A Digital to Analog Converter transmits the digital control input into a continuous signal. The interface board consists of four main stages. The first is the microcontroller $(\mu \mathrm{C})$ stage, which is the AT89C51. The second stage is the $5 \mathrm{~V}$ input Analog to Digital Converted (ADC). The third one is the $10 \mathrm{~V}$ input Analog to Digital Converter. The fourth stage is the $10 \mathrm{~V}$ output Digital to Analog Converter (DAC).

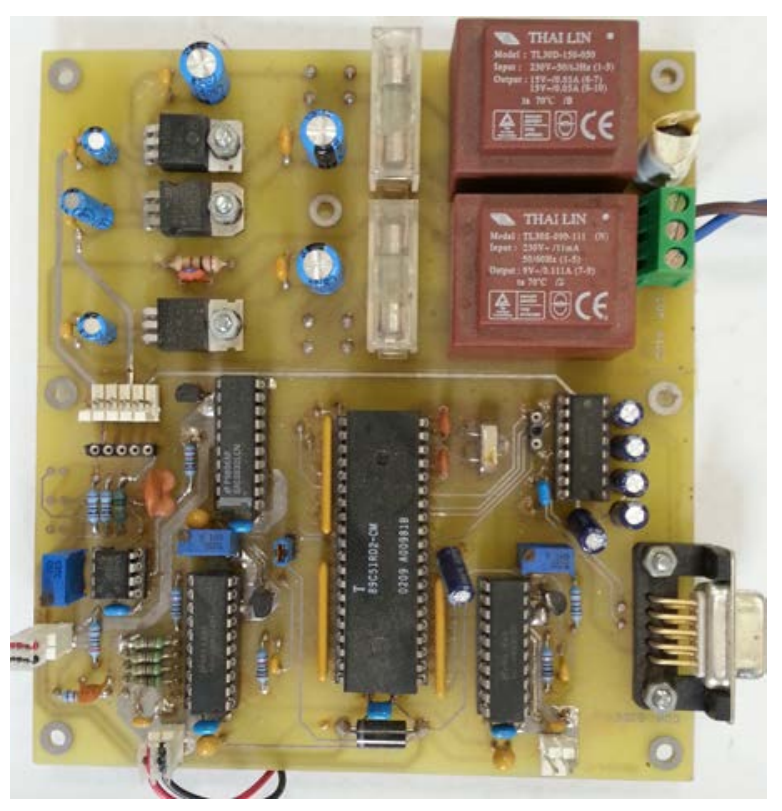

Figure 5. The interface board

The microprocessor, a flash AT89C51, is responsible to synchronize the communication between the sensors and the computer. The serial interface and the control of the converters are being controlled in this stage. The clock frequency is selected to be $11.0592 \mathrm{MHz}$ so it can be divided exactly for the RS-232 serial communication. A circuit breaker, which is shorted on power up, is used for downloading every new program code to the microcontroller. For the second and third design stages, the Analog to Digital Converters, a ADC0803 8 bit $\mu \mathrm{C}$ compatible is used. This is a common ADC, with excellent characteristics. It's a successive approximation A/D converter that uses a potentiometric ladder, as in [10], [11] and [12]. This converter appears as a memory location to the Input-Output ports of the $\mu \mathrm{C}$ and so no interfacing logic is needed. In addition to this, the voltage reference input can be adjusted to allow encoding any smaller analog voltage span to the full 8 bit resolution. Another significant feature is that this converter has an on-chip clock generator and the conversion time is $100 \mu \mathrm{sec}$. For the $10 \mathrm{~V}$ input ADC stage the same converter is used with just a voltage divider added to the input of the converter. The fourth stage of the interface control board is the DAC0830, which is used as a Digital to Analog converter in a voltage switching configuration. In this configuration the ladder is operated as a voltage switching network and not as the standard current switching. 
The reference voltage is connected to one of the current output terminals and the output voltage (Vref) is provided by the normal reference pin of the microcontroller. The converter output is a voltage in the range from $0 \mathrm{~V}$ to $255^{*}$ Vref/256 as a function of the applied digital code. In this configuration the applied reference voltage must be always positive to prevent unacceptable behavior. There is also a dependence of conversion linearity and gain error on the voltage difference between the supply voltage and the voltage applied to the normal current output terminals. This is a result of the voltage drive requirements of the ladder switches. The power supplies voltages needed for the Interface board are $+5 \mathrm{~V},+15 \mathrm{~V}$ and $-15 \mathrm{~V}$. A power supply providing all these voltages is included on the card. If an external power supply is to be used then the card can be further minimized and the part with the power supply equipment can be removed. Then the voltages needed for the interface board can be supplied to it through a power supply connector. Assembling this kind of boards with ADCs and DACs, it is critical to design in a certain way the power lines, especially ground connections, to ensure proper operation.

In this board, special care has been given to this. In addition, it would be useful at this point to highlight the interface controls between the userengineer and the system. The micro-processor provides a user friendly environment for programming, which is illustrated in Figure 6. In that figure, there are three different buttons corresponding to the three terms of the controller gains. There are also buttons to set the piston target position, to start the experimentation timer and the log window that monitors the system's signals. In Visual Basic 6.0 (vb6) is quite simple to build an interface software.

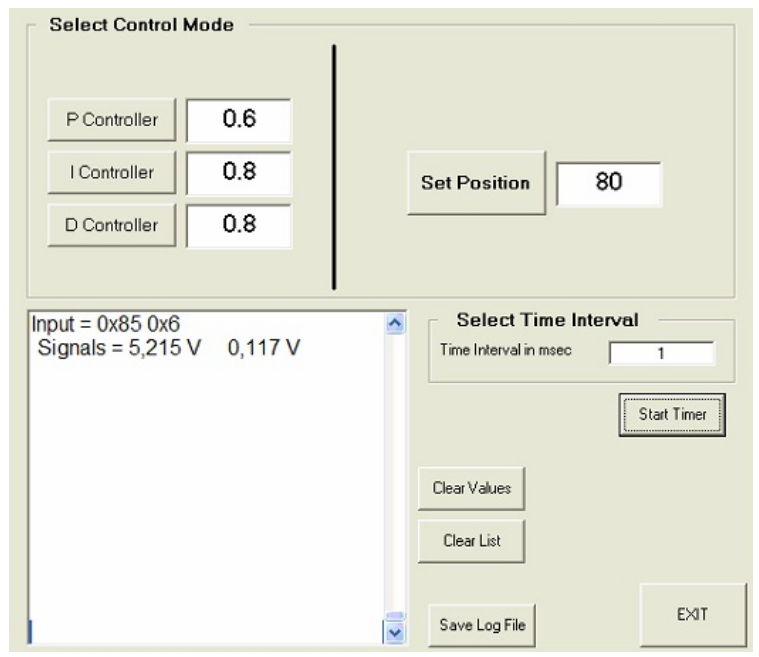

Figure 6. The programming interface panel
The functions needed are few which makes it even easier. This program is build in order to test the interface card's functionality. The interval of the control signal and the PID's parameters can be selected. There is an ability to save out logs in an Excel File where an analysis of them can take place afterwards.

The gains of each parameter and the Desired Position of the system (mm) can be shown then. On the left part a list shows the input and output signal, values presented as hex and volts. The user sets the parameters and the desired position in $\mathrm{mm}$ while by pressing the Start Timer button, the control begins. The Timer stops when the system reaches the desired position or if the user stops the control by pressing the same button. In order to save the information of the response, the button "Save Log File" needs activation and all information is saved in an excel sheet. In the first two lines of this sheet, the Starting Position and the Desired Position are saved. The parameters of PID

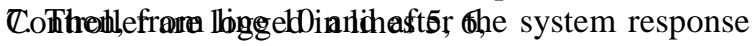
and the control signal are saved. The control signal in $\mathrm{mm}$ is saved in the same column with the previous information. In the next two columns is the response of the plant, in mm and volts. Experiments have an empty column between them. The maximum length of the data logged is 1000 . If the plant has not settled in desired position in $1000 \mathrm{msec}$ then the control automatically stops, and so do the data being logged. Else, the column length depends in the time the user stops the timer (the samples are taken when the timer overflows). The user can save up to ten experiments this way and all following data will be overwritten. The layout of the interface was a custom design that meets the requirements of this specific task. The versatility of the interface design in this project is a helpful tool for an engineer in order to communicate and control multiple dynamic systems.

\section{First Controller performance}

Although the design of the classical threeterm control for nonlinear multivariable systems has been extensively studied in many books and papers, the design procedures for such high order nonlinear systems, like the pneumatic systems, may be complicated and vary from case to case. A simple approach to robust control, and the main topic of this chapter, is the implementation in the system of a classical controller which would automatically choose whether or not all terms of control P, I, D are appropriate for the application. During experimentation we managed to witness that the system operates satisfactory with the use of PD control and that the existing steady state position error was the main unwanted characteristic of the system behavior. The solution to that was to address the I-term control in the controller so that the steady state error would be eliminated. 
The need for retuning three rather than two control gains on one hand and the fact that the overall system behavior became oscillatory under the influence of the I-term, turned us to implement a clever idea of using the Iterm only for beneficiary results. The new technique, which was addressed in the system, is based on "switching" the Integration term 'on' and 'off' according the value of the steady state error. Although the integration term is limiting the steady state error to tiny values close to zero over short periods, it is also producing the unacceptable system response over long time periods of operation. This technique introduces another secondary 'zone' of the steady state error which is placed in the middle of the primary 'zone' of $4 \mathrm{~mm}(+/-2 \mathrm{~mm})$ when the I-term switches on, discussed earlier in this section. The secondary zone of values is, like the primary zone, split into two parts, $0.5 \mathrm{~mm}$ above and $0.5 \mathrm{~mm}$ below the demand piston position respectively. The idea of the new control algorithm is that the I-term is switched on when the error is within the primary zone $(2 \mathrm{~mm}$ above the demand position and $2 \mathrm{~mm}$ below it) but when the error values are very small, i.e., within the secondary zone of values, it switches off. When the system performs without the influence of the Iterm, the behavior is not oscillatory, and therefore if the steady state error remains always in the secondary zone, the response is considered to be acceptable. The new algorithm is based on the generic PID algorithm with this slight modification allowing the existence of the secondary zone and in fact this method provides the privilege to the system of 'deciding', according to the value of steady state, error whether to perform with or without the integration influence. The operation of the system required the implementation of manual retuning, but the undesired oscillations of the system were eliminated. In Figure 7 the system response when this method is applied to it is shown. It is useful to explain at this point some multiple critical comments, which can be recorded from the given plot below. In order to estimate the steady state error, the position axis of the plot is extremely focused around the desired position value $(64 \mathrm{~mm})$. The system responses, as well as the demand position signal, therefore appear noisy. There are three different system responses, test 1 (orange line), test 2 (gray line) and test 3 (light blue line), which are the average curves of ten different experiments each, with the same control gain values and the desired target position is set to the $3 / 4$ of the piston stroke. The system with the new method of the secondary zone of error values performs rather well, there are no oscillations during long time operations (50 sec) as there were before, with the simple PID control and the critical factor of this research project, the position accuracy, is minimized in values between $0.16 \mathrm{~mm}$ and $0.2 \mathrm{~mm}$ of the demand position.

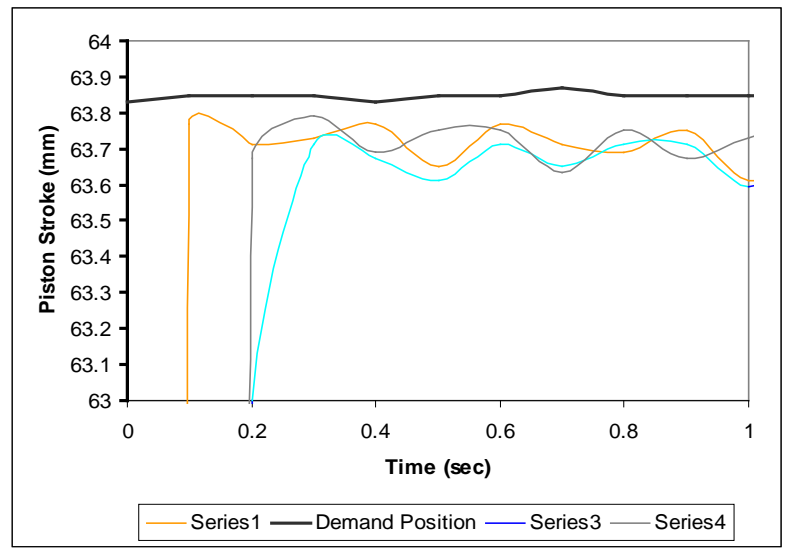

Figure 7. The system performance focused around target position

The piston position percentage accuracy is calculated as the $0.28 \%$ of the overall stroke of the piston, a value that is excellent considering the nature of the system. After all, this new method of control improved the overall system response and the time spent on retuning the redesigned algorithm was worth it for the aim of this project. The experimentation to back-up our results was designed as follows. During 8 hours continuous operations of the system all responses of the $\mathrm{PD}$, PID, Auto-selective ITerm, were recorded. Then, an average response of each one of the techniques was plotted and an illustration of that is nrovided in Figure 8

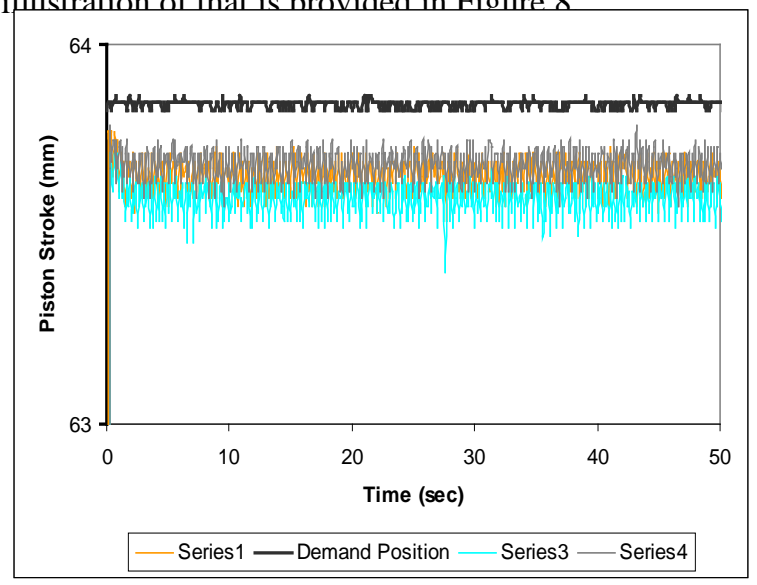

Figure 8 . The system performance

On the other hand, the simulation process took place and outcomes were recorded. In Figure 4 the "S" block which is multiplied with the "I" control term, represents a switch for activating and deactivating that term according to piston position. The same method was followed as the real rig experimentations including all zone restrictions for the I-term control. The result of "S" switch is either 0 outside the position zones, or 1 when the piston is inside them 
respectively. There is a special algorithm running within that block that ensures the switching function of it. With this methodology, the results of the simulation process are shown in the below Figure 9.

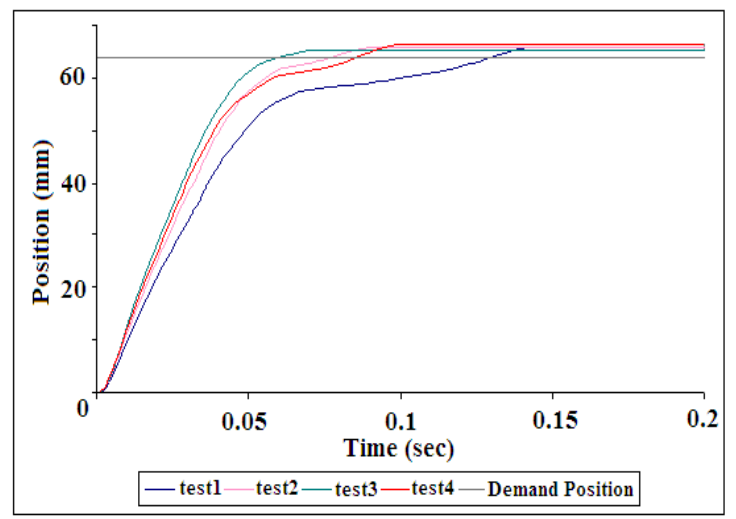

Figure 9. The system performance in simulation

In the same figure, all best achievable system responses for the ultimate $\mathrm{Kp}-\mathrm{Kd}-\mathrm{Ki}$ gain values are provided. The target (demand) position is set to $64 \mathrm{~mm}$ representing the $75 \%$ of the piston effective stroke and the tests were carried out at 4 different supply pressures. The first thing to discuss referring to performance, is an important factor for all dynamic systems, which is the rise time; the simulation process is faster than the real operation and this is understandable considering that in the simulation there are only mathematical equations to be solved and not complex physical phenomena as in the real world. Another example is the supply pressure, which affects the performance of the model in a way like, the higher the pressure is, the faster the system response is and this is something acceptable.

\section{Second Controller Performance}

As mentioned earlier, the second control method which was applied to the system was the fuzzy logic method, considering its promising results in a non-linear system as the pneumatic positioning system, see [13] and therefore was decided to be implemented in the simulation environment.

The control performance of a traditional controller fully depends on the accuracy of a known system dynamic model. The complex pneumatic servodrive positioning process has non-linear and time varying behaviours; thus it is difficult to derive and identify an appropriate dynamic model for traditional controllers. It is very difficult to get an accurate and linearised mathematical model; therefore the fuzzy control technology is applied.In recent years, the control technology has been well developed and has become one of the most successful tools in the industry. In fact, nowadays, there are several programmable logic controller dealers who offer fuzzy control tools in their products. This kind of control has been applied in the industrial world (process and automation) giving an optimal effect. The results of this application have demonstrated that the fuzzy control shows better benefits in comparison with that those offered by other PID controllers. The main advantages of the fuzzy controller are:

- It is not necessary to build a detailed mathematical model. Despite this, in thepresent work, this point is considered bymeans of Bond Graph Technique.

- The fuzzy controllers have a highstrength and a high adjustment.

- They can operate with a high inputnumber.

- They can be adapted easily into non-linear systems.

- The human knowledge can be easilyapplied.

- The process development time isrelatively lower.

The essence of fuzzy logic control is that appropriate linguistic fuzzy rules are chosen, using some decision-making process, from a rule table constructed using human control experience and databases. In the present work the fuzzy rules are established by trial and error with the concept of symmetry. Fuzzy set theory is employed to simulate the logic reasoning of human beings. Fuzzy control has been demonstrated to provide highly satisfactory results in terms of accuracy, repeatability and insensitivity to changes in operating conditions. Classic controls satisfies the requirements for stability, accuracy and rapid response; providing that there is an optimal match between the real values of the system's physical parameters and the values used for control design, and there is no external interference (change in load). Advanced control techniques (e.g. optimum, robust, self tuning) requires highly sophisticated and complex control algorithms if they are to be any effective use. Applying fuzzy control to a continuous pneumatic positioning system is particularly advantageous in terms of simplicity of design and implementation, and thus significantly reduces the time required to develop the entire system. Experience shows that the success of a fuzzy control depends on the level of knowledge concerning the positioner's physical behaviour. Fuzzy logic was originally proposed by Lofti Zadeh in 1965 with the work "Fuzzy sets" and then developed as a tool for manipulating and processing vague information in uncertain conditions. One of the main characteristics of this approach is the element partial membership which allows smooth transitions from one rule to another In this context, the production of the membership functions, i.e., functions that define the membership 
degrees for each input and output of the system is called "fuzzyfication". All fuzzy set representing the crisp (physics) variables related by membership functions are the so called "knowledge basis". The knowledge basis has uncertain information however significant for the system modeling. Although this uncertain is completely solved as the input and output fuzzy sets and the knowledge manipulation strategy are defined. A fuzzy algorithm processes the membership functions for each one of the fuzzy sets and the results are aggregating through instructions or rules, producing the so called "rule basis". Often, in order to establish a truth degree for the rules each fuzzy output is multiplied by an appropriate scale factor. There are basically two types of fuzzy system models differentiating in the ability of representing different kinds of information, i.e, in the form of representing the rule basis, as in [14]. The first include the linguistic models based in collections of IF THEN rules with vague attributes and have fuzzy reasoning. In this type of model, fuzzy quantities are associating with linguistic labels and a fuzzy model is essentially a qualitative expression of the system. The second type of model is based in the TakagiSugeno method. These models are constructed by logic rules which are combination of fuzzy and crisp models. A set of inference rules is adopted to manipulate the knowledge basis. The most used method to represent the human knowledge is through natural language expressions as: IF (antecedent) THEN (consequent).

Since decisions are based on the testing of all of the rules in the inference system, the rules must be combined in some manner in order to make a decision. Aggregation is the process by which the fuzzy sets that represent the outputs of each rule are combined into a single fuzzy set.

One of the most used is the Mamdani implication method for inference in which the aggregated output is:

$\mu_{\mathrm{B}_{\mathrm{a}}^{\mathrm{k}}}(\alpha(\mathrm{i}), \alpha(\mathrm{j}))=\max \left[\min \left[\mu_{\mathrm{A}_{\mathrm{at}}^{\mathrm{k}}}(\alpha(\mathrm{i})), \mu_{\mathrm{A}_{\mathrm{a} 2}^{\mathrm{k}}}(\alpha(\mathrm{j}))\right]\right], \quad$ for $\mathrm{k}=1, \ldots, \mathrm{r}$

where An1k and An2k represent antecedent fuzzy sets, $\mu$ represent membership functions, Bnk represent the consequent fuzzy set for inputs a(i) and $a(j)$. Often the output of fuzzy process must be a scalar quantity and not fuzzy sets. A crisp value for the system output is obtained by the defuzzyfication of the fuzzy output set. In the literature there are some defuzzyfication methods as, for instance, centroid, bisector, middle of maximum (the average of maximum values of the output set), largest of maximum, and smallest of maximum. Perhaps the most popular defuzzification method is the centroid calculation, which returns the center of area under the curve. This was the method used in this work and the crisp value is obtained by the center of area given by the gathering of the output membership functions as:

$$
y^{*}=\frac{\int \mu_{B_{x}^{k}}(y) y d y}{\int \mu_{B_{a}^{k}}(y) d y}
$$

where $\mathrm{y}^{*}$ is the value obtained by the defuzzyficationand Bnk are the consequent fuzzy sets. For comparisons reasons, a mathematical block sequel was designed in Matlab Simulink in order to test the system performance under a fuzzy logic controller. All above information was adopted during experimentation and the system block diagram is illustrated in Figure 10. The block 'Pneumatic Plant' contains all system dynamics like discussed earlier in the paper and the block 'Fuzzy Controller' contains the control algorithm that produces the necessary signal for the system to operate.

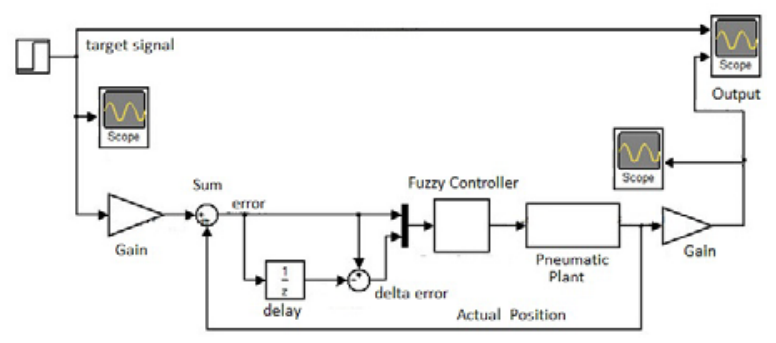

Figure 10. The block diagram of the second system

During a significant number of tests and frequent re-tunings of the fuzzy controller the ultimate output was then recorded and is provided in Figure 11. It is obvious, that the system although stabilizes without steady state error, its behavior is oscillatory at the beginning of the stroke. This unacceptable behavior is present at all edges of a square pulse input. The response time of the system is pretty small i.e. less than a second. It worth to pay attention that this output is recorded with the minimum parameterization and tuning of the fuzzy controller and it looks as though with more, for example, membership functions involved, the outcome will get even better in terms of improving oscillations.

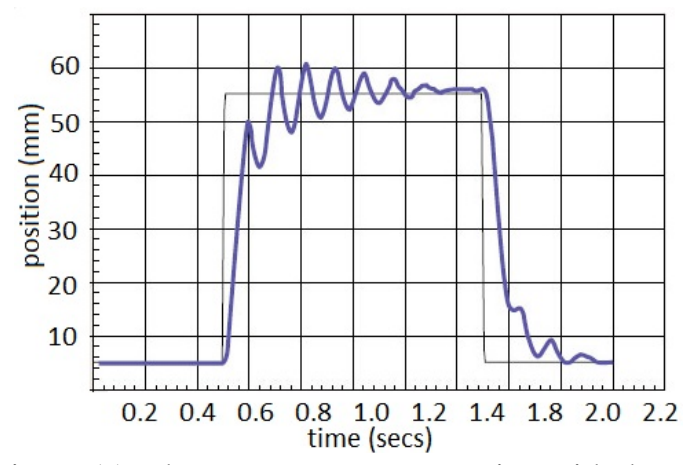

Figure 11. The system output operating with the second controller 


\section{Conclusions and Further Work}

The system control outcome under the first control technique, although acceptable, still left space for a different control method to be adopted, like, an Intelligent Control Method. The initial challenge to investigate if the implementation of such a method would require an upgrade in the hardware of this specific interface card was answered negatively and this fact is the most important advantage of the board. The micro controller source code, as the software part, is the only bit that needs to be re-designed in order to adopt the new control method algorithm. This fact proves the versatility of the interface board designed and built for the requirements of the present research project. Furthermore the ability of these electrical components is proven to be stable and capable enough to host multiple control tasks and monitor their performance successfully. In addition, the position error of the model operation in simulation (Fig 7), with this PID controller method applied to it, is calculated between $1.3 \mathrm{~mm}$ to $2.5 \mathrm{~mm}$, which finally gives $2.2 \%$ position accuracy. This value is large and is a factor that verifies the need for deeper investigation in the controller performance. Although the overshoot is constant and there are no sudden response spikes, not many conclusions can be derived from the current simulation process of the PID position control implementation. The same simulation system was robust enough to host another control technique and keep following up the real experimentation's methods. Given this as a fact, the fuzzy logic control method was adopted in the system and all recordings led to the system output of Figure 11. In contrast with the classical controller, the intelligent presents a more oscillatory performance, an almost same response time but a significantly decrease in steady state error (thus position accuracy) of 1.2 $\mathrm{mm}$.

In conclusion, this research study introduced a interface board to control a highly nonlinear dynamic positioning system with a "smart" control method. At the same time a simulation model of the whole system was built to verify the control results of the real system. Both can be considered as helpful tools for further investigation and a joint robust platform for testing various modern control methods. Additionally, it is important to make evident the great potential that fuzzy logic has to offer, such as the no need for the mathematical model. However high system knowledge is necessary to improve the performance of the controlled servodrive. Another point to consider is also the easy integration of the fuzzy logic in traditional control algorithm, as it is shown in this work. Another advantage that the fuzzy logic offers is that an autotuning algorithm can be applied to the system, by the means of this reasoning. In this way, the system can learn the which control parameters to take automatically and this is the next research project to be undertaken in the close future.

\section{References}

[1] Richard E.,Scavarda, S.,1996, Comparison Between Linear and Nonlinear Control of an Electropneumatic Servodrive ,Journal of Dynamic Systems, Measurement and Control,Vol.118,pp.245-118.

[2] Jihog Wang, Junsheng Pu, Philip Moore, “Accurate Position Control of Servo Pneumatic Actuator Systems", Control Engineering Practice, 1999, pp. 699-706

[3] J. E. Bobrow, B.W. McDonell, "Modeling, Identification and Control of a Pneumatically Actuated, Force Controllable Robot”, draft Thesis 1990, California University,USA.

[4] Saravanan Rajendran and Robert W.Bolton, 'Position Control of a Servopneumatic Actuator using Fuzzy Compensation', "Proceedings of the 2003 American Society for Engineering Education Annual Conference \& Exposition

[5] Paulo Roberto Barbosa and Paulo Seleghim Jr., 'On the Application of Fuzzy Logic Control in Pneumatic Conveying systems', Journal of the Brazilian Neural Network Society, Vol. 9, Iss.4, pp. 256-265, 2011.

[6] Osama Olaby, Xavier Brun, Sylvie Sesmat, Tanneguy Redarce and Eric Bideaux, 'Characterization And Modeling Of A Proportional Valve For Control Synthesis', Proceedings of the 6th JFPS International Symposium on Fluid Power, Tsukuba 2005, November 7-10, 2005.

[7] Richer E. and Hurmuzlu Y., 'A High Performance Force Actuator System Part 1 Nonlinear Mathematical Model', ASME Journals of Dynamics Systems Measurement and Control, 2000, Vol. 122, No3, pp. 416425

[8] Belforte G., Mattiazzo G., Mauro S., "Design criteria for flow proportional control valves”, Proceedings of sixth triennual international symposium on Fluid control, Measurement and Visualization, Flucome 2000, August 13-17, Sherbrooke, Canada.

[9] Papoutsidakis M.G., Chamilothoris G.E, Dailami F., Larsen N. and Pipe A., (2005) "Accurate control of a pneumatic system using an innovative fuzzy gainscheduling pattern”, International Academy of Sciences, 6th International Conference on Enformatika, System Science and Engineering, Budapest, Hungary.

[10] Khol, R., editor, "Electrical \& Electronics Reference Issue,” Machine Design, Vol 57, No 12, May 30, 1985.

[11] Benjamin C. Kuo, Digital Control Systems, Second Edition, New York: Oxford University Press. 
[12] J. Proakis and D. Manolakis, Digital Signal Processing: Principles, Algorithms, and Applications, New York: Macmillan Publishing Company, 1992.

[13] Hipòlit Moreno Llagostera, 'Control of a Pneumatic Servosystem Using Fuzzy Logic', Proc. of 1st FPNI-PhD Symp. Hamburg 2000, pp. 189-201

[14] Behrouz Najjari, S. Masoud Barakati, Ali Mohammadi , Muhammad J. Futohi and Muhammad Bostanian, 'Position control of an electro-pneumatic system based on PWM technique and FLC', Elsevier ISA Transactions 53 (2014) 647-657

\section{Acknowledgements}

All authors would like to thank the Piraeus University of Applied Sciences for providing the necessary equipment and funding to undertake this research work. 